\title{
A note on the Penetration of Semi-infinite Metallic Targets Struck by Long Rods at High Velocities
}

\author{
Y. He \\ CAS Key Laboratory for Mechanical Behavior and Design of \\ Materials \\ University of Science and Technology of China \\ Hefei, Anhui, China \\ hexiaoyu@mail.ustc.edu.cn
}

\author{
H.M. Wen \\ CAS Key Laboratory for Mechanical Behavior and Design of \\ Materials \\ University of Science and Technology of China \\ Hefei, Anhui, China \\ hmwen@ustc.edu.cn
}

\begin{abstract}
Analytical equations are presented herein to predict the penetration of semi-infinite metallic targets struck normally by long rods at high velocities. The analysis is based on energy balance where the kinetic energy loss of a long rod is related to the energy dissipated by the plastic deformations in the target, the energy consumed by the long-rod itself and the kinetic energy remaining in the ejected rod debris. The present analytical equation is found to be in good agreement with the experimental data for a wide range of impact velocities.
\end{abstract}

\section{Keywords-penetration, long rod, semi-infinite metallic target}

\section{INTRODUCTION}

Much effort has been directed towards the research of long rod penetrators penetrating targets for more than half a century. Significant amount of terminal ballistic data and numerical simulation results were obtained for the combination of penetrators and targets made of various materials[1]-3]. Overviews of long rod penetration were presented by Zook et al.[4], Goldsmith[5] and Orphal[6].

A one-dimensional model proposed independently by Alekseevskii[7] and Tate[8] has become the standard reference for long rod penetration into semi-infinite metallic targets and various modifications and extensions of the Alekseevskii-Tate model have been made by many authors[9]-[20].

\section{PENETRATION DEPTH}

Consider a unitary long rod of length $L_{0}$ penetrates into a semi-infinite target at impact velocity $v$, the initial kinetic energy of the long rod can then be expressed as

$$
E_{k}=\frac{1}{2} \pi r^{2} \rho_{p} L_{0} v^{2}
$$

where $r$ and $\rho_{p}$ are the radius and density of the long rod, respectively.

To a first approximation, it is assumed that the kinetic energy loss of the penetrator is mainly related to the energy dissipated by plastic deformations of the target, the energy associated with the crushing/erosion of the rod itself and the kinetic energy remaining in the ejected rod debris. The energy for crushing the penetrator $\left(E_{r o d}\right)$ may be expressed as

$$
E_{\text {rod }}=\pi r^{2} Y_{p} L_{0}
$$

where $Y_{p}$ is the dynamic strength of penetrator material. The energy dissipated by plastic deformations in the target $\left(E_{\text {target }}\right)$ may be approximated as

$$
E_{\text {target }}=\pi R_{\text {crater }}^{2} P S,
$$

where $P$ is the total depth of penetration in the target, $S$ is the work expended per unit volume of the crater[21] which can be taken as the static resistive pressure derived from cavity expansion approximation and $R_{\text {crater }}$ is the crater diameter in the semi-infinite target which can be evaluated as[19]

$$
\frac{R_{\text {crater }}}{r}=\sqrt{\frac{Y_{p}+\rho_{p}(\varphi+1)(v-u)^{2}}{S}},
$$

where

$$
\varphi=\sqrt{1-\frac{2 Y_{p}}{\rho_{p}(v-u)^{2}}} .
$$

The kinetic energy remaining in the ejected rod debris can be expressed as

$$
E_{\text {debris }}=\frac{1}{2} \pi r^{2} \rho_{p} L_{0} v_{e}^{2}
$$

where $v_{e}$ is the velocity of the ejected rod debris with opposite direction to impact velocity determined by[19]

$$
V_{\mathrm{e}}=\varphi(v-u)-u
$$

Hence, from the energy balance one obtains

$$
E_{k}=E_{\text {rod }}+E_{t \arg e t}+E_{\text {debris }}
$$

Substituting (1-7) into (8) and rearranging gives

$$
\frac{P}{L_{0}}=\frac{1 / 2 \rho_{p} v^{2}-1 / 2 \rho_{p}\left(\sqrt{(v-u)^{2}-2 Y_{p} / \rho_{p}}-u\right)^{2}-Y_{p}}{Y_{p}+\rho_{p}(v-u)\left(\sqrt{(v-u)^{2}-2 Y_{p} / \rho_{p}}+(v-u)\right)}
$$

or

$$
\frac{P}{L_{0}}=\frac{I-\left(\sqrt{I(1-u / v)^{2}-2}-\sqrt{I} u / v\right)^{2}-2}{2+2(1-u / v)\left(\sqrt{I^{2}(1-u / v)^{2}-2 I}+I(1-u / v)\right)},
$$

where $I=\frac{\rho_{p} v^{2}}{Y_{p}}$ and $u$ is the penetration velocity which can be determined by the following equations, viz. [18] 


$$
\begin{aligned}
\frac{1}{2} \rho_{t}[u-\delta(u)]^{2}+S+C \rho_{t} \delta(u)^{2} & =\frac{1}{2} \rho_{p}(v-u)^{2}+Y_{p} \text { for } \\
u & \geq U_{F 0}
\end{aligned}
$$

or

$$
S+C \rho_{t} u^{2}=\frac{1}{2} \rho_{p}(v-u)^{2}+Y_{p} \text { for } u \leq U_{F 0}
$$

where

$$
\delta(u)=U_{F 0} \exp \left[-\left(\frac{u-U_{F 0}}{n U_{F 0}}\right)^{2}\right], n \geq 2 \sqrt{C}
$$

and $\rho_{t}$ is target density, $C$ is the constant of the dynamic resistive pressure and for incompressible materials, $C=3 / 2$, $U_{F 0}$ is the critical penetration velocity above which a flow region appears in the target near the penetrator-target interface, otherwise there are only plastic and elastic regions and $U_{F O}=\sqrt{H E L / \rho_{t}}$ where HEL is the Hugoniot Elastic Limit of the target material[18].

If the impact velocity is allowed to go to very high or the dynamic strength of rod and target material are allowed to go to zero, the dimensionless number $I$ is allowed to become very large and then (10) gives the same penetration depth with hydrodynamic theory.

\section{COMPARISONS AND DISCUSSION}

Figure 1 shows comparison of (10) with the experimental data for the penetration into semi-infinite armor steel targets struck by tungsten alloy long rods with $L / D=23[22]$. In the theoretical calculation, the dynamic strength of the tungsten alloy is taken be $2082.5 \mathrm{MPa}[18]$ and the density is 17300 $\mathrm{kg} / \mathrm{m}^{3}$. The material properties of the armor steel targets used in the calculation are given in Table 1. It is clear from Figure 1 that (10) is in good agreement with the experimental data[22] over a wide range of impact velocities. The discrepancy between (10) and the experimental data for impact velocities higher than $3000 \mathrm{~m} / \mathrm{s}$ may be due to the so-called "after-flow" effect which has not been taken into account in the present formulation. It is also can be seen in Fig. 1 that the penetration depth from (10) approaches an asymptote predicted by hydrodynamic theory at high impact velocities.

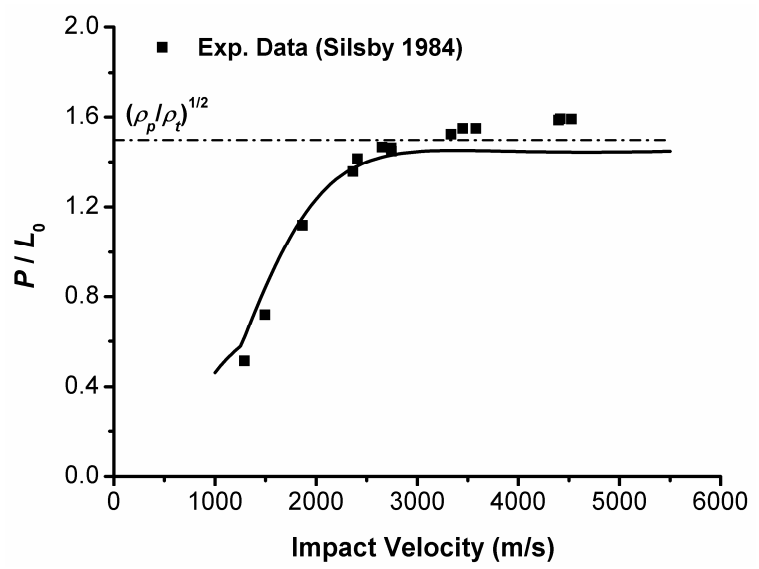

\begin{tabular}{|c|c|c|c|c|c|c|c|c|}
\hline $\begin{array}{c}\text { Target } \\
\text { Materials }\end{array}$ & $\underset{\left(\mathrm{kg} / \mathrm{m}^{3}\right)}{\rho}$ & $\begin{array}{c}E_{1} \\
(\mathrm{GPa})\end{array}$ & $\begin{array}{c}E_{2} \\
(\mathbf{M P a})\end{array}$ & $\lambda$ & $\begin{array}{c}Y_{0} \\
(\mathrm{MPa})\end{array}$ & $\begin{array}{c}Y \\
\text { (MPa) }\end{array}$ & $\begin{array}{l}\text { HEL } \\
\text { (MPa) }\end{array}$ & $\begin{array}{c}S \\
\text { (MPa) }\end{array}$ \\
\hline $\begin{array}{l}\text { Armor } \\
\text { Steel[18] }\end{array}$ & 7850 & 205 & 177.2 & 0.3 & -- & 1130 & 1925 & 4395.2 \\
\hline $\begin{array}{l}\text { GerArmor } \\
\text { Steel[18] }\end{array}$ & 7850 & 205 & 500 & 0.3 & -- & 988.6 & 1730.1 & 4262.9 \\
\hline RHA[23] & 7850 & 205 & 177.2 & 0.3 & -- & 1370 & 2397.5 & 5242 \\
\hline
\end{tabular}

Figure 1. Comparison of equation (10) with the experimental data[22] for semi-infinite armor steel targets impacted by tungsten alloy long rods

TABLE I. MATERIAL PROPERTIES OF THE TARGETS EXAMINED.

Fig. 2 shows comparison of (10) with the experimental data for Ger Armor Steel targets struck by C100W1 steel long rod penetrators with $L / D=10[1]$. In the theoretical calculation, the yield stress of C110W1 was given by Hohler and Stilp as 762 $\mathrm{MPa}(\mathrm{BHN} 230)[1]$. The dynamic strength of $\mathrm{C} 110 \mathrm{~W} 1$ is taken to be $1334 \mathrm{MPa}[19]$. The other material properties of Ger Armor Steel are also listed in Table 1. It can be seen from Fig. 2 that (10) is in good agreement with the test data for impact velocities greater than $1500 \mathrm{~m} / \mathrm{s}$ approximately and for impact velocities less than $1500 \mathrm{~m} / \mathrm{s}$ the depth of penetration is a little bit overpredicted by (10) compared to the experimental results.

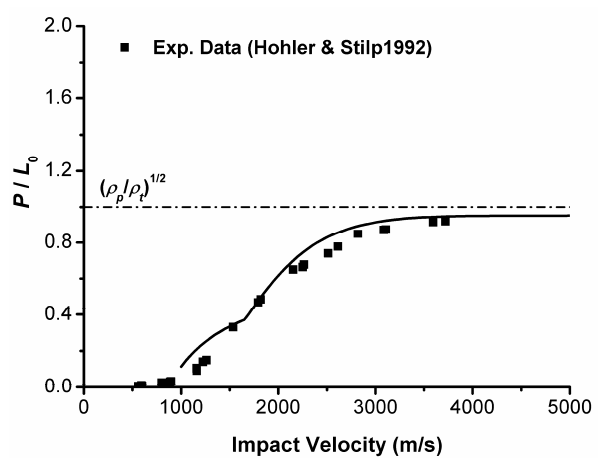

Figure 2. Comparison of equation (10) with the experimental data[1] for semi-infinite armor steel targets impacted by $\mathrm{C} 110 \mathrm{~W} 1$ steel long rods. 
Equation (10) can also be applied to jacketed rod penetration when co-erosion occurs so long as $Y_{p}$ and $\rho$ are replaced with $Y_{e q}$ and $\rho_{e q}$ which are determined by the following equations[20]

$$
\left\{\begin{array}{l}
Y_{e q}==Y_{j}+\left(Y_{c}-Y_{j}\right) \frac{r_{c 0}^{2}}{r_{j 0}^{2}} \\
\rho_{e q}=\rho_{j}+\left(\rho_{c}-\rho_{j}\right) \frac{r_{c 0}^{2}}{r_{j 0}^{2}}
\end{array}\right.
$$

where $r_{c 0}$ and $r_{j 0}$ are core and jacket radii respectively, $\rho_{c}$ and $\rho_{j}$ are respective core and jacket material densities, $Y_{c}$ and $Y_{j}$ are the dynamic strengths of core and jacket materials, respectively. The relationship of $v-u$ for a jacketed rod penetration when co-erosion occurs can also be determined by those of unitary long rod penetration of the same core material from (11) and (12) where $Y_{p}=Y_{c}$ and $\rho_{p}=\rho_{c}$.

Figure 3 shows comparison of (10) with the experimental data reported by Cullis et al [23] for EN24 steel jacketed tungsten alloy long-rod penetrators. The dynamic strength of the core and jacket material were taken to be $3.5 \mathrm{GPa}$ and 1.49 GPa by WEN HeMing, HE Yu et al[20]. The RHA(Rolled Homogeneous Armour) target yield stress (i.e. Y in Table 1) is $1.37 \mathrm{GPa}[23]$ and the other constants are taken to be the same as given in Table 1. It can be seen from Figure 3 that good agreement is obtained between (10) and the test data.

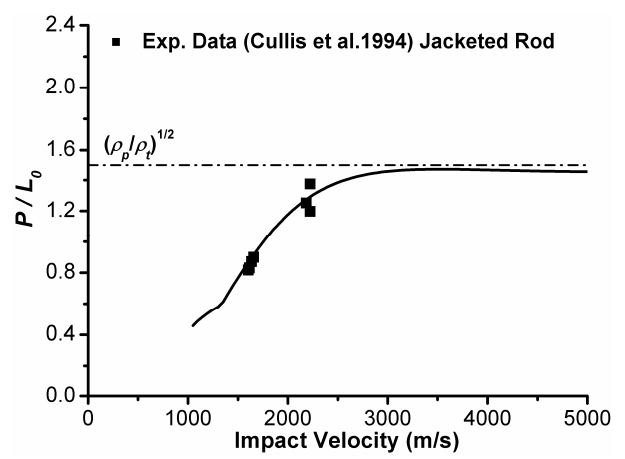

Figure 3. Comparison of equation (10) with the experimental data for EN24 steel jacketed tungsten alloy long rods and tungsten unitary long rods penetrating semi-infinite armor steel targets [23]

\section{CONCLUSIONS}

Analytical equations have been given in this paper to predict the penetration of semi-infinite metallic targets struck normally by high speed long rods. The analysis is based on energy balance where the kinetic energy of a long rod is equated to the energy dissipated by the plastic deformations in the target, the energy consumed by the long-rod itself and the kinetic energy remaining in the ejected rod debris. It is shown that the present analytical equation is in good agreement with the experimental data for a wide range of impact velocities.

\section{REFERENCES}

[1] Anderson Jr CE, Morris BL, Littlefield DL. A penetration mechanics database. SwRI Report 3593/001, Southwest Research Institute, San Antonio, TX(1992).
[2] Hohler V, Stilp AJ. Hypervelocity impact of rod projectiles with L/D from 1 to 32. International Journal of Impact Engineering. 1987; 5: 323 331 .

[3] Stilp AJ, Hohler V. Aeroballistics and impact physics research at EMI an historical overview. International Journal of Impact Engineering. 1995; 17: 785-805

[4] Zook JA, Frank K, Silsby GF. Terminal ballistics test and analysis guidelines for the penetration branch. BRL-MR-3960, U.S. Army Ballistic Research Laboratory, Aberdeen Proving Ground, MD, 1992.

[5] Goldsmith W. Non-ideal projectile impact on targets. International Journal of Impact Engineering. 1999; 22: 95-395.

[6] Orphal DL. Explosions and impacts. International Journal of Impact Engineering. 2006; 33: 496-545.

[7] Alekseevskii VP. Penetration of a rod into a target at high velocity. Combustion, Explosion and Shock Waves. 1966; 2: 63-66.

[8] Tate A. A theory for the deceleration of long rods after impact. Journal of the Mechanics and Physics of Solids. 1967; 15: 387-399.

[9] Rosenberg Z. On the hydrodynamic theory of long-rod penetration. International Journal of Impact Engineering. 1990; 10: 483-486.

[10] Jones SE, Gillis PP, Foster JC. On the penetration of semi-infinite targets by long rods. Journal of the Mechanics and Physics of Solids. 1987; 35(1): 121-131.

[11] Wang P, Jones SE. An elementary theory of one-dimensional rod penetration using a new estimate for pressure. International Journal of Impact Engineering. 1996; 18(3): 265-279.

[12] Grace FI. Nonsteady penetration of long rods into semi-infinite targets. International Journal of Impact Engineering. 1993; 14: 303-314.

[13] Walker JD, Anderson Jr CE. A time-dependent model for long-rod penetration. International Journal of Impact Engineering. 1995; 16(1): $19-48$.

[14] Rubin MB, Yarin AL. A generalized formula for the penetration depth of a deformable projectile. International Journal of Impact Engineering. 2002; 27: 387-398

[15] Walters WP, Segletes SB. An exact solution of the long rod penetration equations. International Journal of Impact Engineering. 1991; 11(2): 225-231.

[16] Segletes SB, Walters WP. Extensions to the exact solution of the longrod penetration/erosion equations. International Journal of Impact Engineering. 2003; 28: 363-376.

[17] Walker JD. Hypervelocity penetration modeling: Momentum VS. energy and energy transfer mechanisms. International Journal of Impact Engineering. 2001; 26: 809-822.

[18] Lan B, Wen HM. Alekseevskii-Tate revisited: an extension to the modified hydrodynamic theory of long-rod penetration. Sci. China, Ser, E. 2010, 53(5): 1364-1373.

[19] Wen HM, He Y, Lan B. Analytical model for cratering of semi-infinite metal targets by long rod penetration. Sci. China, Tech. Sci., 2010, 53: 3189-3196.

[20] Wen HM, He Y, Lan B. A combined numerical and theoretical study on the penetration of a jacketed rod into semi-infinite target. International Journal of Impact Engineering. 2011, 38:1001-1010.

[21] Hill. R. Cavitation and the influence of headshape in attack of thick targets by non-deforming projectiles. Journal of the Mechanics and Physics of Solids, 1980; 28:249-263.

[22] Silsby GF. Penetration of semi-infinite steel targets by tungsten rods at 1.3 to $4.5 \mathrm{~km} / \mathrm{s}$. Proceeding of the Eighth International Symposium on Ballistics, TB/31-35, Orlando, Florida, 1984.

[23] Cullis IG, Lynch NJ. Hydrocode and experimental analysis of scale size jacketed KE projectiles. Proc.14th Int. Symp. on Ballistics Vol TB-7, Quebec, Canada. 1994:271-280. 